\author{
Military Technical College \\ Kobry Elkobbah, \\ Cairo, Egypt \\ May 29-31,2012
}

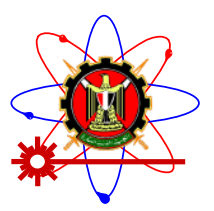
$6^{\text {th }}$ International Conference on Mathematics and Engineering Physics (ICMEP-6)

\title{
Simple Food Chain in Chemostat When the Predator Produces Unaffected Toxin
}

Ashraf A. Moniem

Akhbar El-Yom Academy, 6 October City, Giza (12573), Egypt.

In this paper, Simple food chain in chemostat when the predator produces unaffected toxin is considered. This inhibitor is not lethal to neither prey nor nutrient and results in decrease of growth rate of the predator at some cost to its reproductive abilities. A Lyapunov function in the study of the global stability of a predator-free steady state is considered. Local and global stability of other steady states, persistence analysis, as well as numerical simulations are also presented.

Key wards: food chain - toxin - chemostat - prey - predator - Lyapunov function.

\section{1- Introduction}

The chemostat is a laboratory apparatus used for the continuous culture of microorganisms. It can be used to study competition between different populations of microorganism or between preys and predators, and has the advantage that the parameters are readily measurable. The monograph of Smith and Waltman (8) has various mathematical methods for analyzing chemostat models. Recently, the inhibitor has been introduced in the models for two competitors in a chemostat, and many authors have studied those models (see $(1,2,3,4,5,6$ and 7)).

In this paper, we consider a model of simple food chain in chemostat when the predator produces unaffected inhibitor. This inhibitor is not lethal to neither prey nor nutrient and results in the decrease of growth rate of the predator at some cost to its reproductive abilities.

This paper is organized as follows: In the next section, the model is presented and some simplifications. Section 3, deals with the existence and local stability of steady states. In section 4, we shall provide global analysis, including global stability of the boundary steady states and persistence analysis. Discussion, comments and numerical simulation are found in final section.

\section{2- The model}

The interest equations are

$$
\begin{aligned}
s^{\prime}(t) & =\left(s^{0}-s(t)\right) D-\frac{1}{\gamma_{1}} f_{1}(s(t)) x(t), \\
x^{\prime}(t) & =x(t)\left(f_{1}(s(t))-D\right)-\frac{1}{\gamma_{2}} f_{2}(x(t)) y(t), \\
y^{\prime}(t) & =y(t)\left((1-k) f_{2}(x(t))-D\right), \\
p^{\prime}(t) & =k y(t) f_{2}(x(t))-D p, \\
& 0<s(0), \quad 0<x(o), \quad 0<y(0), \quad 0<p(0) .
\end{aligned}
$$


Military Technical College

Kobry Elkobbah,

Cairo, Egypt

May 29-31,2012

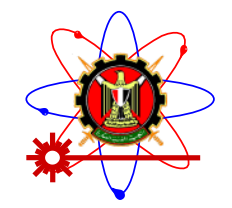

$6^{\text {th }}$ International Conference on Mathematics and Engineering Physics (ICMEP-6)

Where $s(t), x(t), y(t)$ and $p(t)$ are the concentration of the nutrient, prey, predator and inhibitor at time $t$, respectively. $s^{0}$ Denotes the input concentration of the nutrient, $D$ denotes the washout rate. $f_{1}(s(t))=\frac{m_{1} s(t)}{a_{1}+s(t)}$ and $f_{2}(x(t))=\frac{m_{2} x(t)}{a_{2}+x(t)}$ where $m_{i}, i=1,2$, the maximal growth rates, $a_{i}, i=1,2$, the Michaelis- Menten constants and $\gamma_{i}, i=1,2$, the Yield constants. The constant fraction $k \in(0,1)$ is the potential growth due to inhibitor growth (see (3) for description the physical meaning of $k$ ).

For scaling, let

$$
\begin{aligned}
& \bar{s}=\frac{s}{s^{0}}, \quad \bar{x}=\frac{x}{\gamma_{1} s^{0}}, \quad \bar{y}=\frac{y}{\gamma_{1} \gamma_{2} s^{0}}, \quad \bar{t}=D t, \\
& \bar{p}=\frac{p}{\gamma_{1} \gamma_{2} s^{0}}, \quad \bar{m}_{i}=\frac{m_{i}}{D}, i=1,2, \quad \bar{a}_{1}=\frac{a_{1}}{s^{0}}, \bar{a}_{2}=\frac{a_{2}}{\gamma_{1} s^{0}} .
\end{aligned}
$$

Substitute into (2.1) and then drop the bars, the model becomes

$$
\begin{aligned}
& s^{\prime}=1-s-f_{1}(s) x, \\
& x^{\prime}=x\left(f_{1}(s)-1\right)-f_{2}(x) y, \\
& y^{\prime}=y\left((1-k) f_{2}(x)-1\right), \\
& p^{\prime}=k y f_{2}(x)-p .
\end{aligned}
$$

\section{3- Existence and local stability}

Let $T=s+x+y+p$, then $T^{\prime}=1-T$, or $\lim _{t \rightarrow \infty} \sup T(t)=1$.

Since each component is non-negative, the system (2.2) is dissipative and thus, has a compact, global attractor. To simplify (2.2), let $z=p-\frac{k y}{1-k}$, we find that the system (2.2) is taken the form,

$$
\begin{aligned}
& s^{\prime}=1-s-f_{1}(s) x, \\
& x^{\prime}=x\left(f_{1}(s)-1\right)-f_{2}(x) y, \\
& y^{\prime}=y\left((1-k) f_{2}(x)-1\right), \\
& z^{\prime}=-z .
\end{aligned}
$$

Clearly $z(t) \rightarrow 0$ as $t \rightarrow \infty$, so the system (3.1) may be viewed as an asymptotically autonomous system with limiting system

$$
\begin{aligned}
& s^{\prime}=1-s-f_{1}(s) x, \\
& x^{\prime}=x\left(f_{1}(s)-1\right)-f_{2}(x) y, \\
& y^{\prime}=y\left((1-k) f_{2}(x)-1\right) .
\end{aligned}
$$


Military Technical College

Kobry Elkobbah,

Cairo, Egypt

May 29-31,2012 $6^{\text {th }}$ International Conference

on Mathematics and

Engineering Physics

(ICMEP-6)

It is easy to show that (3.2) in positive cone. As a consequence, the global attractor of (3.1) lies in the set $z=0$, where (3.2) is satisfied. When the analysis of (3.2) is completed, the work of Thieme (10), relates the corresponding dynamics of (3.1) and (3.2), and hence of (2.2). We will show that all solutions of (3.2) tend to rest points and hence, using Thieme (9), so do those of (2.2).

The equilibrium point $E_{0}=(1,0,0)$, always exists. If $1<f_{1}(1)$, then there is an equilibrium of (3.2) of the form $E_{1}=\left(\lambda_{s}, 1-\lambda_{s}, 0\right)$, where $\lambda_{s}$, is the unique solution of $f_{1}\left(\lambda_{s}\right)-1=0$.

Similarly, if $\frac{1}{1-k}<f_{2}(1)$, there is an equilibrium of the form

$E_{2}=\left(s^{*}, \lambda_{x}, \lambda_{x}(1-k)\left(f_{1}\left(s^{*}\right)-1\right)\right)$, where $s^{*}$, is the unique value of $s$, such that

$1-s-\lambda_{x} f_{1}(s)=0$, and $\lambda_{x}$, is the unique solution of $(1-k) f_{2}(x)-1=0$.

We now discuss the existence of steady state. The washout steady state $E_{0}$, always exists. A predator-free steady state $E_{1}$, exists when $\lambda_{s}<1$. For the interior steady state $E_{2}$, exists when $\lambda_{s}<1$, and $\lambda_{s}+\lambda_{x}<1$. Note that $H(s)=1-s-\lambda_{x} f_{1}(s)$, is decreasing function in $s$, with $0<H(0)=1, H\left(s^{*}\right)=0$, and $H\left(\lambda_{s}\right)=1-\lambda_{s}-\lambda_{x}$. So $\lambda_{s}<s^{*}$, if and only if $\lambda_{s}+\lambda_{x}<1$.

Next theorem will be investigated the local stability of these steady state by finding the eigenvalues of the associated Jacobian matrices.

\section{Theorem 3.1}

If $1<\lambda_{s}$ then only $E_{0}$ exists and $E_{0}$ is locally asymptotically stable. If $\lambda_{s}<1$ and $1<\lambda_{s}+\lambda_{x}$, then only $E_{0}$ and $E_{1}$ exist, $E_{0}$ is unstable, and $E_{1}$ is locally asymptotically stable. If $\lambda_{s}<1$ and $\lambda_{s}+\lambda_{x}<1$ then $E_{0}, E_{1}, E_{2}$ exist, and $E_{0}, E_{1}$, are unstable. $E_{2}$, is locally asymptotically stable if $0<a_{1}$ and $a_{3}<a_{1} a_{2} \quad\left(a_{i}, i=1,2,3\right.$ will be defined in proof)

\section{Proof}

The variation matrix of (3.2) is taken the form

$$
\left[\begin{array}{lll}
-1-x f_{1}^{\prime}(s) & -f_{1}(s) & 0 \\
x f_{1}^{\prime}(s) & f_{1}(s)-1-y f_{2}^{\prime}(x) & -f_{2}(x) \\
0 & (1-k) y f_{2}^{\prime}(x) & (1-k) f_{2}(x)-1
\end{array}\right]
$$

At $(1,0,0$,$) this is$

$$
\left[\begin{array}{lll}
-1 & -f_{1}(1) & 0 \\
0 & f_{1}(1)-1 & 0 \\
0 & 0 & -1
\end{array}\right]
$$


Military Technical College

Kobry Elkobbah,

Cairo, Egypt

May 29-31,2012 $6^{\text {th }}$ International Conference

on Mathematics and

Engineering Physics

(ICMEP-6)

The eigenvalues are on the diagonal and the washout steady state will be locally asymptotically stable if and only if $f_{1}(1)-1<0$, or $1<\lambda_{s}$.

At $\left(\lambda_{s}, 1-\lambda_{s}, 0\right)$ the variation matrix becomes

$$
\left[\begin{array}{lll}
-1-\left(1-\lambda_{s}\right) f_{1}^{\prime}\left(\lambda_{s}\right) & -f_{1}\left(\lambda_{s}\right) & 0 \\
\left(1-\lambda_{s}\right) f_{1}^{\prime}\left(\lambda_{s}\right) & 0 & -f_{2}\left(1-\lambda_{s}\right) \\
0 & 0 & (1-k) f_{2}\left(1-\lambda_{s}\right)-1
\end{array}\right]
$$

The determinant of the upper left- hand $2 \times 2$ matrix is positive and its trace is negative, so its eigevalues have negative real parts. The third eigenvalue is $(1-k) f_{2}\left(1-\lambda_{s}\right)-1$. Therefore the predator - free steady state is asymptotically stable if and only if $(1-k) f_{2}\left(1-\lambda_{s}\right)-1<0$, or $1<\lambda_{s}+\lambda_{x}$.

The variation matrix at $E_{2}$, takes the form

$$
\left[\begin{array}{lll}
-1-\lambda_{x} f_{1}^{\prime}\left(s^{*}\right) & -f_{1}\left(s^{*}\right) & 0 \\
\lambda_{x} f_{1}^{\prime}\left(s^{*}\right) & \left(f_{1}\left(s^{*}\right)-1\right)\left(1-\lambda_{x}(1-k) f_{2}^{\prime}\left(\lambda_{x}\right)\right) & -\frac{1}{1-k} \\
0 & \lambda_{x}(1-k)\left(f_{1}\left(s^{*}\right)-1\right) f_{2}^{\prime}\left(\lambda_{x}\right) & 0
\end{array}\right]
$$

The eigenvalues of $E_{2}$, satisfy $\alpha^{3}+a_{1} \alpha^{2}+a_{2} \alpha+a_{3}=0$, where

$$
\begin{aligned}
& \begin{aligned}
a_{1}= & 1+\lambda_{x} f_{1}^{\prime}\left(s^{*}\right)+\left((1-k) \lambda_{x} f_{2}^{\prime}\left(\lambda_{x}\right)-1\right)\left(f_{1}\left(s^{*}\right)-1\right), \\
a_{2}= & \lambda_{x} f_{1}\left(s^{*}\right) f_{1}^{\prime}\left(s^{*}\right)+(1-k) \lambda_{x} f_{2}^{\prime}\left(\lambda_{x}\right)\left(f_{1}\left(s^{*}\right)-1\right) \\
& +\left(1+\lambda_{x} f_{1}^{\prime}\left(s^{*}\right)\right)\left((1-k) \lambda_{x} f_{2}^{\prime}\left(\lambda_{x}\right)-1\right)\left(f_{1}\left(s^{*}\right)-1\right), \\
a_{3}= & (1-k) \lambda_{x} f_{2}^{\prime}\left(\lambda_{x}\right)\left(1+\lambda_{x} f_{1}^{\prime}\left(s^{*}\right)\right)\left(f_{1}\left(s^{*}\right)-1\right) .
\end{aligned}
\end{aligned}
$$

Clearly $0<a_{3}$, so from the Routh-Hurwitz criterion, $E_{2}$ is locally asymptotically stable if and only if $0<a_{1}$ and $a_{3}<a_{1} a_{2}$.

\section{4- Global analysis}

\section{Theorem 4.1}

For $1<\lambda_{s}$ and for large $t$, all solutions of (3.2) tends to $E_{0}$.

\section{Proof}

For $1<\lambda_{s}$ and for large $t$, we get $s(t)<1$ and $f_{1}(1)<1$. Therefore, the second equation of (3.2) gives $x(t)<e^{-\left(1-f_{1}(1)\right) t}$, which imply to $\lim _{t \rightarrow \infty} x(t)=0$. The third equation of 
Military Technical College

Kobry Elkobbah,

Cairo, Egypt

May 29-31,2012

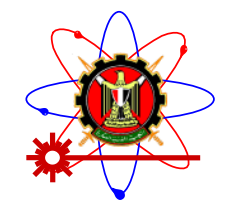

$6^{\text {th }}$ International Conference on Mathematics and Engineering Physics (ICMEP-6)

(3.2) becomes $y=e^{-t}$, which leads to $\lim _{t \rightarrow \infty} y(t)=0$. The first equation of (3.2) has a solution $s=1+($ cons $\tan t) e^{-t} \rightarrow 1$ as $t \rightarrow \infty$.

\section{Theorem 4.2}

If $\lambda_{s}<1, \quad 1<\lambda_{s}+\lambda_{x}$ and for large $t$, then all solutions of (3.2) tend to $E_{1}$.

\section{Proof}

Let

$$
\eta=1+\frac{\left(1-\lambda_{s}-x\right) f_{2}(x)}{\left(1-(1-k) f_{2}(x)\right) x}, \quad \text { for } \quad 0<x \leq 1-\lambda_{s}
$$

and

$$
\beta=\frac{\eta}{f_{2}(x)}\left((1-k) f_{2}(x)-1\right) \quad \text { for } \quad \lambda_{x} \leq x .
$$

Let $C(u)$ be a continuously differentiable function and $C^{\prime}(u)$ be defined by

$$
C^{\prime}(u)= \begin{cases}0 & \text { if } u \leq 1-\lambda_{s}, \\ \beta \frac{\left(u+\lambda_{s}-1\right)}{\left(\lambda_{s}+\lambda_{x}-1\right)} & \text { if } 1-\lambda_{s}<u<\lambda_{x}, \\ \beta & \text { if } \lambda_{x} \leq u .\end{cases}
$$

Note that $C^{\prime}(u)$ is linear on $\left[1-\lambda_{s}, \lambda_{x}\right]$. We may construct a Lyapunov function as follows:

$V(s, x, y)=\int_{\lambda_{s}}^{s} \frac{\left(1-\lambda_{s}\right)\left(f_{1}(\xi)-1\right)}{(1-\xi)} d \xi+x-*^{*} \ln (x)+\eta y+C(x)$.

on the set $\Psi=\{(s, x, y): 0<s+x+y<1\}$, where ${ }^{*} x=1-\lambda_{s}$.

Differentiate (4.4) with respect to time $t$, we obtain

$$
\begin{aligned}
\dot{V} & =x\left(f_{1}(s)-1\right)\left[1+C^{\prime}(x)-\frac{\left(1-\lambda_{s}\right) f_{1}(s)}{(1-s)}\right] \\
& +y\left[\frac{f_{2}(x)}{x}\left(1-\lambda_{s}-x\right)+\eta\left[(1-k) f_{2}(x)-1\right]-f_{2}(x) C^{\prime}(x)\right]
\end{aligned}
$$

First the term $x\left(f_{1}(s)-1\right)\left[1-\frac{\left(1-\lambda_{s}\right) f_{1}(s)}{(1-s)}\right]$ is nonpositive for $0<s<1$ and equal zero for $s \in[0,1)$ if and only if $s=\lambda_{s}$ or $x=0$. Since $C^{\prime}(x)=0$ for $\lambda_{s} \leq s$ and $C^{\prime}(u) \geq 0$ for $u \geq 0$, then the term $x\left(f_{1}(s)-1\right) C^{\prime}(x)$ is nonpositive for $s \in[0,1)$.

Define 
Military Technical College

Kobry Elkobbah,

Cairo, Egypt

May 29-31,2012

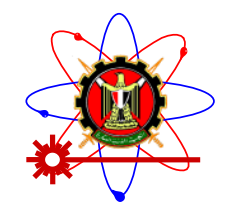

$6^{\text {th }}$ International Conference on Mathematics and Engineering Physics (ICMEP-6)

$$
h(s, x, y)=\left[\frac{f_{2}(x)}{x}\left(1-\lambda_{s}-x\right)+\eta\left[(1-k) f_{2}(x)-1\right]-f_{2}(x) C^{\prime}(x)\right] .
$$

If $0<x \leq 1-\lambda_{s}$,

Then

And

$$
\left[(1-k) f_{2}(x)-1\right] \leq 0, \quad 0 \leq\left[\frac{f_{2}(x)}{x}\left(1-\lambda_{s}-x\right)\right.
$$

$$
0 \leq f_{2}(x) C^{\prime}(x)
$$

Use the definition of $\eta$, we find that $h(s, x, y) \leq 0$.

If $1-\lambda_{s}<x<\lambda_{x}$, then all terms of $h(s, x, y)$ are nonpositive.

If $\lambda_{x} \leq x$, then $C^{\prime}(x)=\beta$ and use definition of $\beta$ and $\eta$, we find that $h(s, x, y)$ will be nonpositive and the second term of $\dot{V}$ equal zero at $y=0$. therefore $\dot{V}$ is nonpositive on $\Psi$. A largest invariant subset $M$ of $\phi=\{(s, x, y) \in \Psi: \dot{V}=0\}$ such that $\dot{V}=0$ at $s=\lambda_{s}$ or $x=0$ and $y=0$. More further, $V$ is bounded above, any point of the form $(s, 0,0)$ can not be in the $\omega$ - limit set $\Omega$ of any solution initiating in the interior of $R_{+}^{3}$. $\left(\lambda_{s}, x, 0\right) \in M$, implies that $s=\lambda_{s}$ and from the first equation of (3.2), we get $x=1-\lambda_{s}$. Therefore $M=\left\{E_{1}\right\}$. This complete the proof.

\section{Theorem 4.3}

If $\lambda_{s}<1$ and $\lambda_{s}+\lambda_{x}<1$, then the system ( 3.2) is uniformly persistence.

\section{Proof}

$$
\begin{aligned}
& \text { Let } Y_{1}=\{(s, x, y): s \in[0,1], \quad x, y \in(0,1]\}, \\
& Y_{2} \text { represents sx-plane }: 0 \leq s, x \leq 1, \\
& Y_{3} \text { represents sy-plane }: 0 \leq s, y \leq 1, \\
& \text { and } Y=Y_{2} \cup Y_{3} .
\end{aligned}
$$

We want to show that $Y$ is a uniformly strong repeller for $Y_{1}$. Since $E_{0}$ and $E_{1}$ are the only steady states in $Y . E_{0}$ is saddle in $R^{3}$ and its stable manifold is $\{(s, 0, y): 0 \leq y\}$. Also, $E_{1}$ is saddle in $R^{3}$ and its stable manifold is $\{(s, x, 0): 0<x\}$. Then, they are weak reppelers for $Y_{1}$. The stable manifold structures of $E_{0}$ and $E_{1}$ imply that they are not cyclically chained to each other on the boundary $Y$. Therefore $Y$ is a uniform strong repeller for $Y_{1}$ (see proposition (1.2) of Thieme (10)).

So, there are $\varepsilon_{1}>0$ and $\varepsilon_{2}>0$ such that $\liminf _{t \rightarrow \infty} x(t)>\varepsilon_{1}$ and $\liminf _{t \rightarrow \infty} y(t)>\varepsilon_{2}$ with $\varepsilon_{1}$ and $\varepsilon_{2}$ are not depending on the initial values in $Y_{1}$. By proposition (2.2) of Thieme (10) to the first equation of (3.2) yields that there is $\varepsilon_{3}>0: \liminf _{t \rightarrow \infty} s(t)>\varepsilon_{3}$ with $\varepsilon_{3}$ is not depending on the initial values in $Y_{1}$. Proof is completed. 
Military Technical College

Kobry Elkobbah,

Cairo, Egypt

May 29-31,2012 $6^{\text {th }}$ International Conference on Mathematics and Engineering Physics (ICMEP-6)

\section{Conclusion and numerical simulation}

In this work, we consider a food chain with one prey and one predator in the chemostat, when the predator produces unaffected toxin. This inhibitor is not lethal to neither prey nor nutrient and results in decrease of growth rate of the predator at some cost to its reproductive abilities. We found that the washout steady state is the global attractor, if it is the only steady state and $\lambda_{s}>1$. When the washout and the predator free steady states are the only steady states, we found that $E_{0}$ is unstable and $E_{1}$ is locally asymptotically stable. $E_{1}$ is global attractor by constructing a Lyapunov function under condition that $\lambda_{s}<1$ and $\lambda_{s}+\lambda_{x}>1$. We also showed that $E_{2}$ is locally asymptotically stable if and only if $0<a_{1}$ and $a_{3}<a_{1} a_{2} . E_{2}$ exists in the sense that the system is uniformly persistent.

We find by numerical simulation that its dynamical behavior is complex. Eight iterative examples are presented here to show the influence of increasing the parameter $k$ on the dynamical behavior. In all examples, parameters values of (3.2) are as follows: $(s(0), x(0), y(0))=(0.1,0.7,0.8), m_{1}=4.0, m_{2}=5.0, a_{1}=0.6, a_{2}=0.5$.

When $k \in] 0,0.4\left[\right.$, the solution appears to approach a periodic solution. So, $E_{0}, E_{1}$ and $E_{2}$ lose their stability ( see figs. 1a, 1b, 2a, 2b, 3a and 3b ). These oscillatory solutions appear to be the results of Hopf bifurcations. The numerical simulation shows that the system (3.2) has an attracting limit cycle.

At $k \in\left[0.4,6.5\right.$ [, the solution approaches a positive steady state. Both $E_{0}$ and $E_{1}$ are unstable and $E_{2}$ is globally asymptotically stable ( see figs. 4a, 4b, 5a, 5b, 6a, 6b, 7a and 7b ). For $k \in\left[6.5,1\right.$ [, the solution approaches the predator-free steady state. $E_{0}$ is unstable and $E_{1}$ is globally asymptotically stable ( see figs. $8 \mathrm{a}$ and $8 \mathrm{~b}$ ). All left figures plot in time courses and all right figurers plot the trajectory in $(s, x, y)$ space.
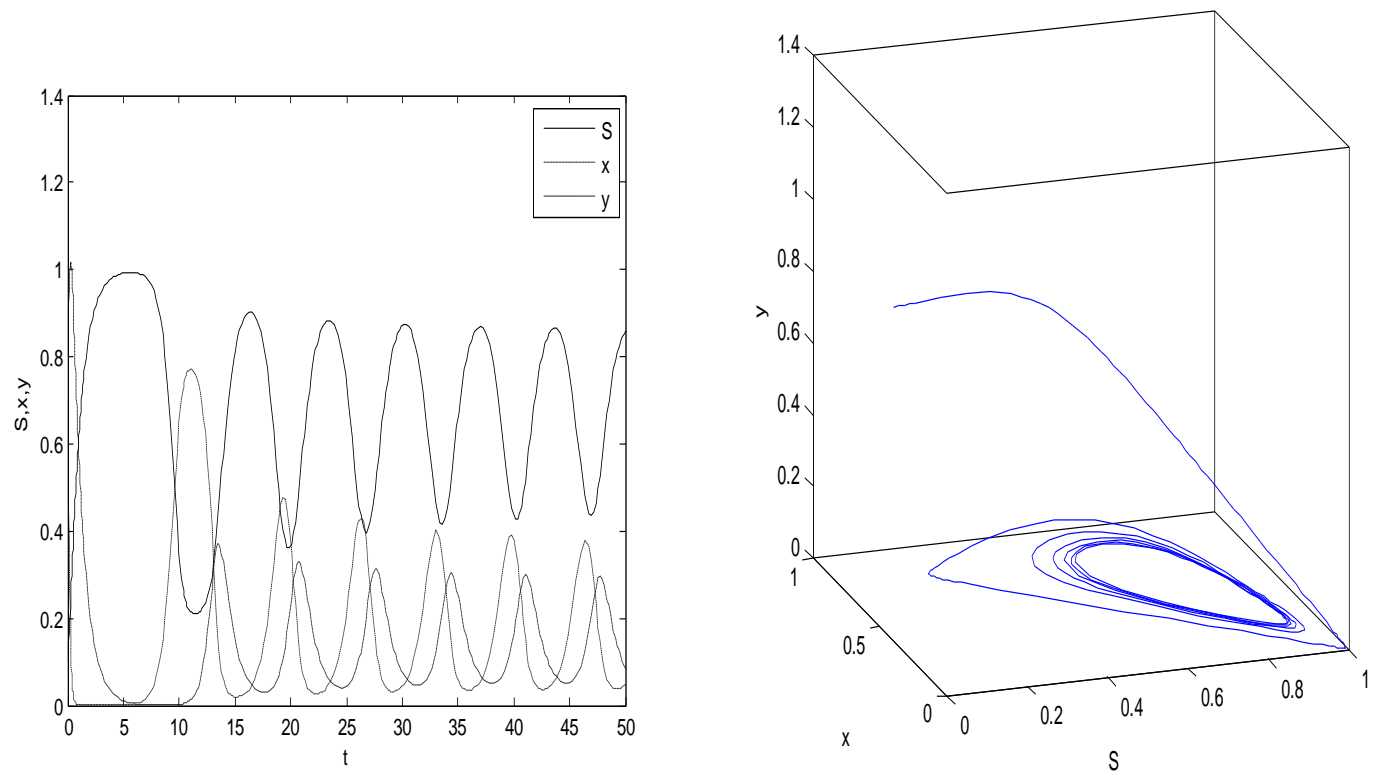
Military Technical College

Kobry Elkobbah,

Cairo, Egypt

May 29-31,2012

Fig.(1a). $k=0.1$

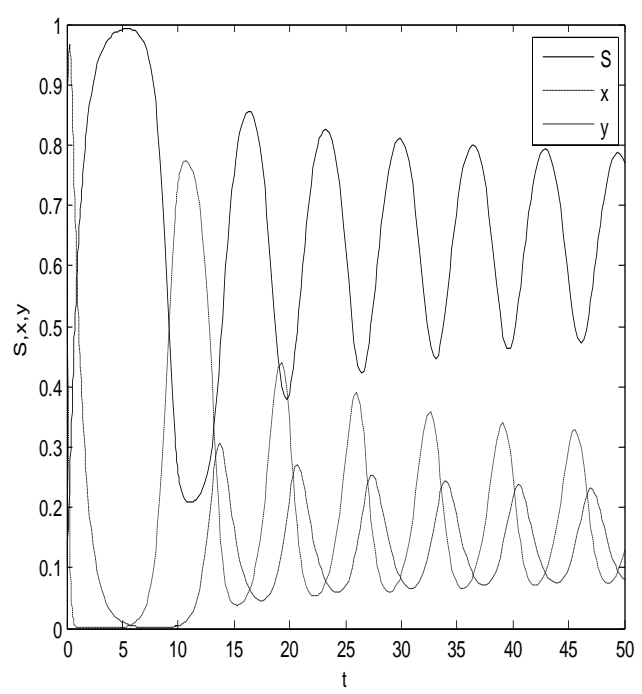

Fig.(2a). $k=0.2$

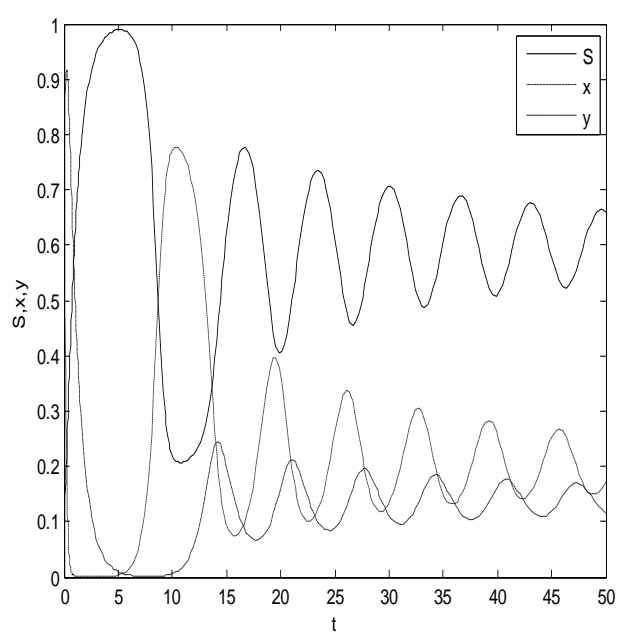

Fig.(3a). $k=0.3$ $6^{\text {th }}$ International Conference on Mathematics and Engineering Physics (ICMEP-6)

Fig.(1b). $k=0.1$

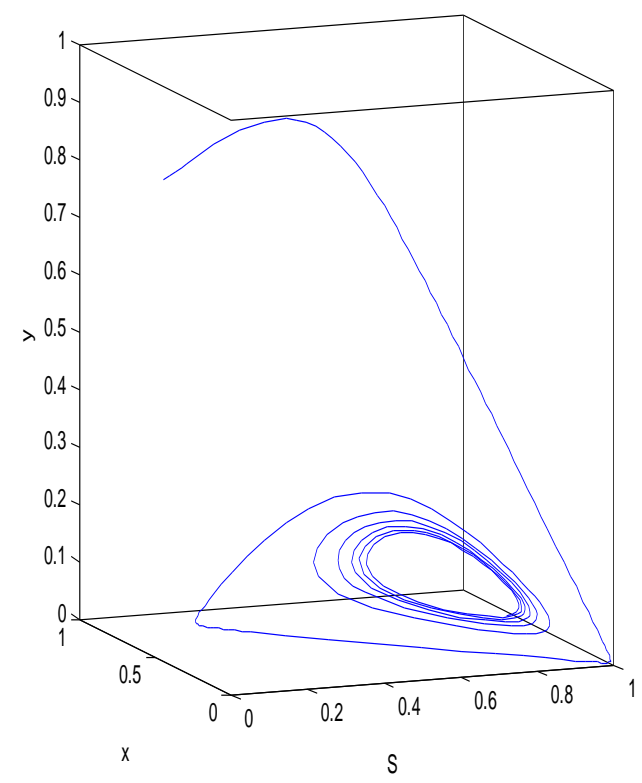

Fig.(2b). $k=0.2$

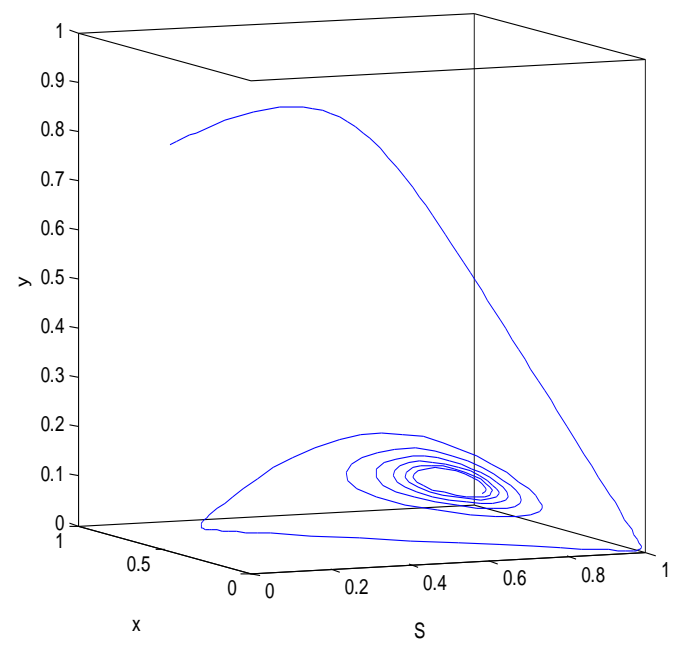

Fig.(3b). $\mathrm{k}=0.3$ 
Military Technical College

Kobry Elkobbah,

Cairo, Egypt

May 29-31,2012

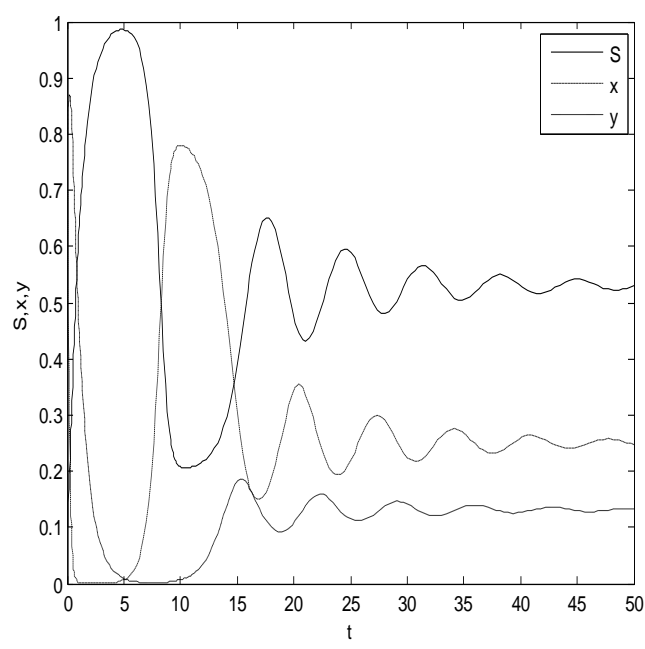

Fig.(4a). $k=0.4$

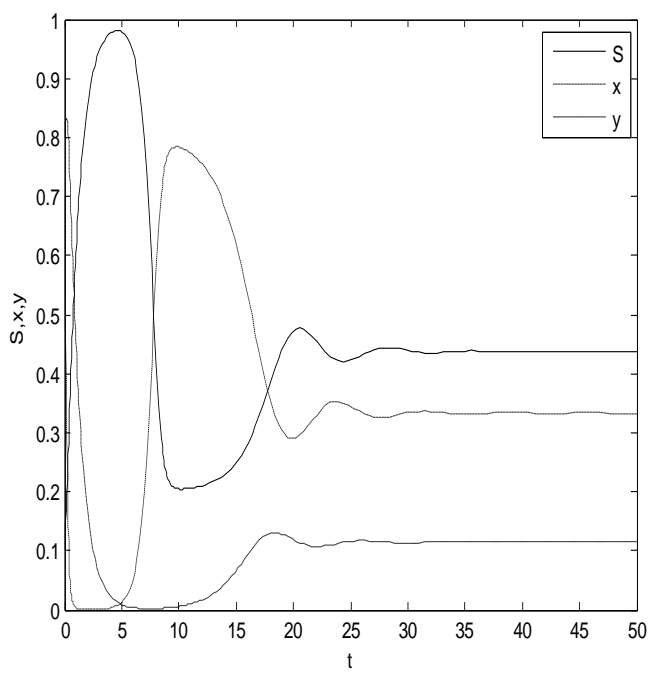

Fig.(5a). $k=0.5$ $6^{\text {th }}$ International Conference on Mathematics and Engineering Physics (ICMEP-6)

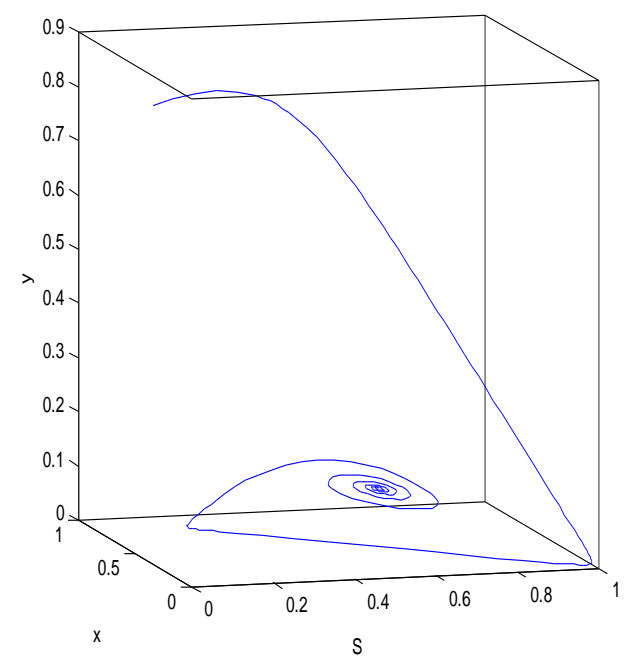

Fig.(4b). $k=0.4$

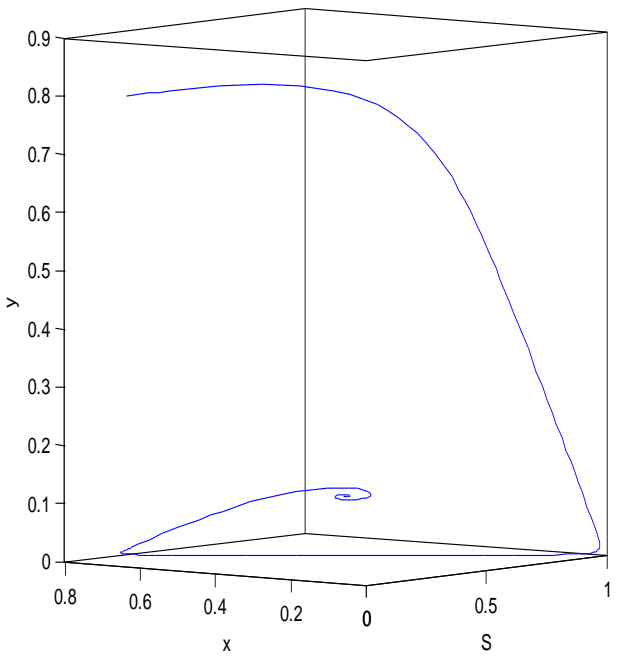

Fig.(5b). $k=0.5$ 
Military Technical College

Kobry Elkobbah,

Cairo, Egypt

May 29-31,2012

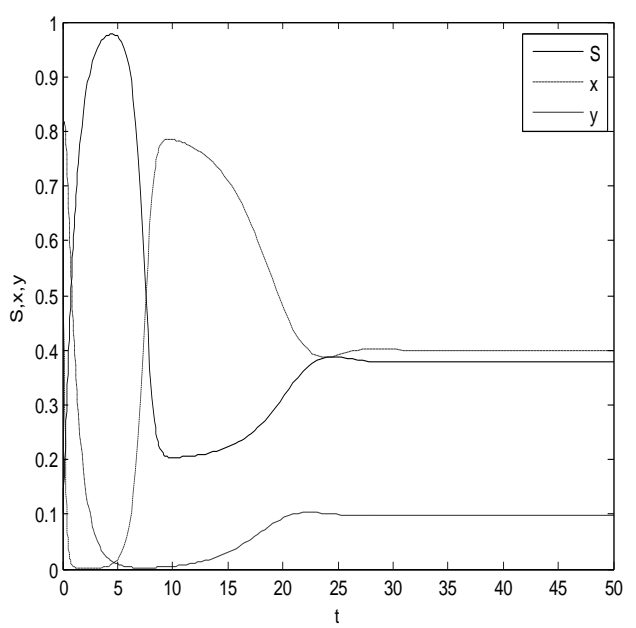

Fig.(6a). $k=0.55$

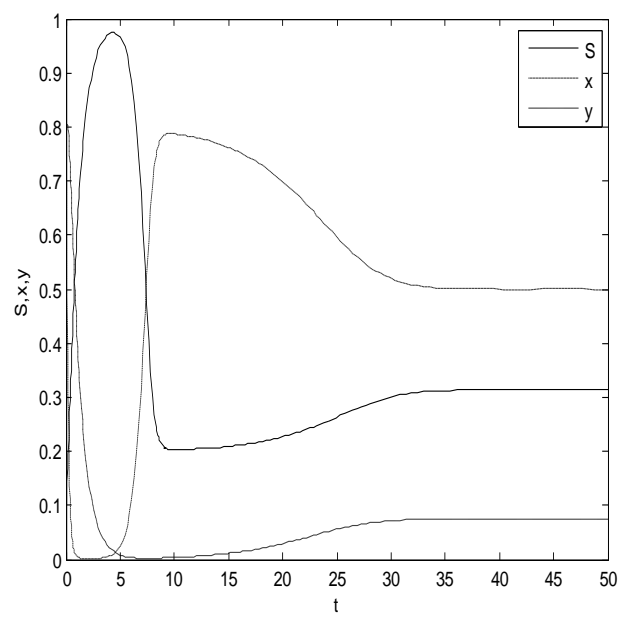

Fig.(7a). $k=0.6$ $6^{\text {th }}$ International Conference on Mathematics and Engineering Physics (ICMEP-6)

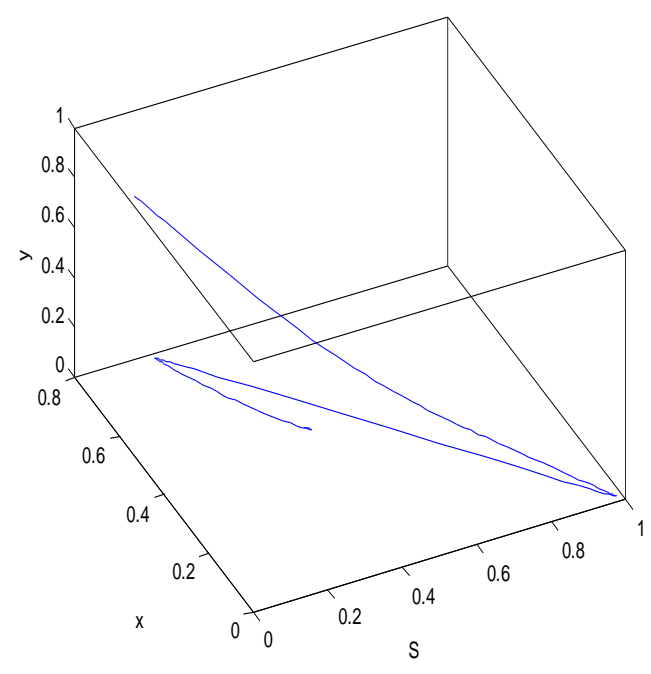

Fig.(6b). $k=0.55$

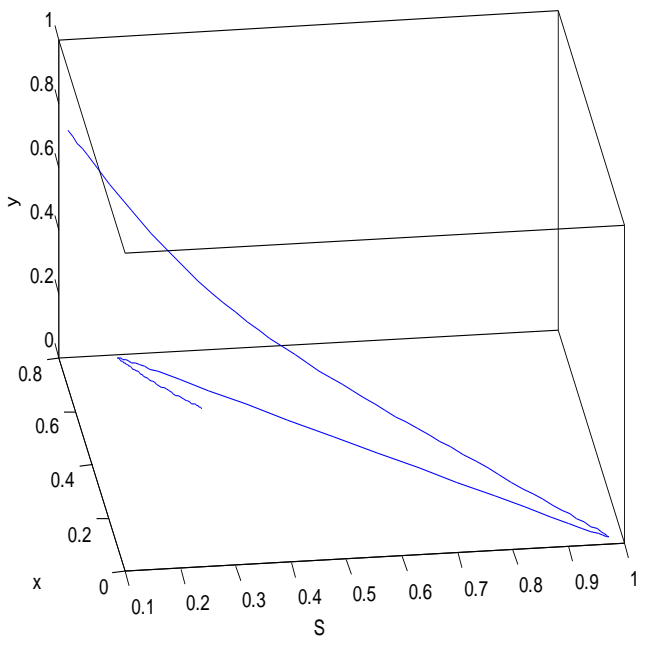

Fig.(7b). $k=0.6$ 


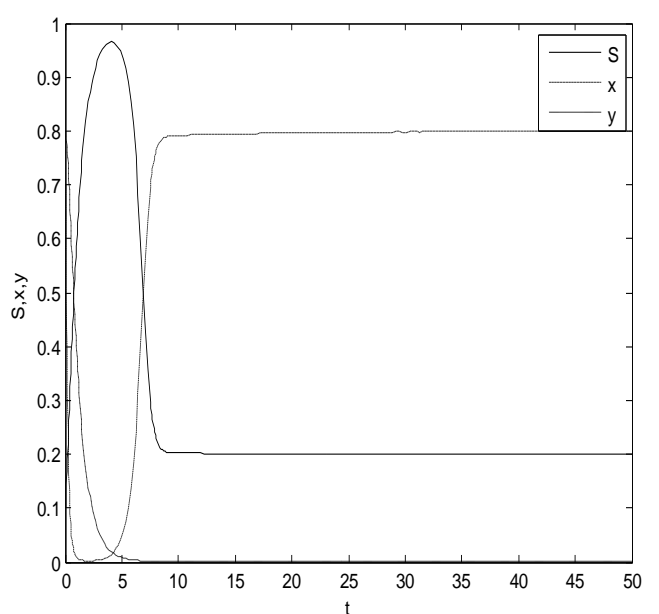

Fig.(8a). $k=0.7$

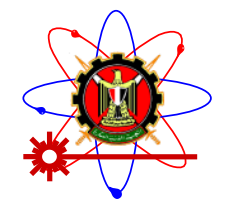

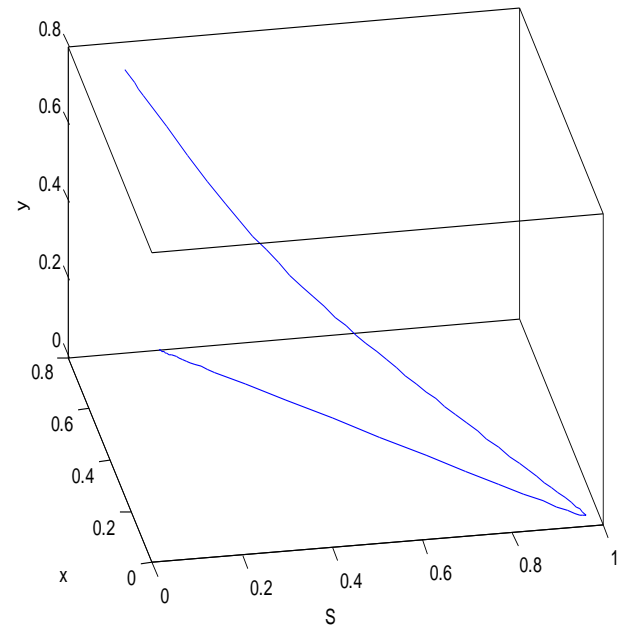

Fig.(8b). $\mathrm{k}=0.7$

\section{REFERENCES}

[1] J. P. Braselton and P. Waltman, A COMPETITION MODEL WITH DYNAMICALLY ALLOCATED INHIBITOR PRODUCTION. Math.Biosci. 173(2001) 55-84.

[2] S. B. Hsu and P. Waltman, ANALYSIS OF A MODEL OF TWO COMPETITORS IN A CHEMOSTAT WITH AN EXTERNAL INHIBITOR. SIAM J. Appl. Math. 52(1992) 528-540.

[3] S. B. Hsu and P. Waltman, COMPETITION IN THE CHEMOSTAT WHEN ONE COMPETITOR PRODUCES A TOXIN. Japan J. Indust. Appl. Math. 15(1998) 471-490.

[4] S. B. Hsu, Ting-kung Luo, and P. Waltman, COMPETITION BETWEEN PLASMID-BEARING AND PLASMIDFREE ORGANISMS IN A CHEMOSTAT WITH AN INHIBITOR. J. Math. Boil. 34(1995) 225-238.

[5] S. B. Hsu, Yen-Sheng Li, and P. Waltman, COMPETITION IN THE PRESENCE OF A LETHAL EXTERNAL INHIBITOR. Math. Biosci. 176(2000) 177-199.

[6] R. E. Lenski and S. Hattingh, COEXISTENCE OF TWO COMPETITORS ON ONE RESOURCE AND ONE INHIBITOR; A CHEMOSTAT MODEL BASED ON BACTERIA AND ANTIBIOTICS. J. Theoret. Biol. 122(1986) 83-93.

[7] J. Li, Z. Feng, J. Zhang and J. Lou, A COMPETITION MODEL OF THE CHEMOSTAT WITH AN EXTERNAL INHIBITOR. Math. Boisci. and Eng. 3(2006) 111-123.

[8] H. Smith, P. Waltman , THE THEORY OF CHEMOSTAT. Cambridge University Press, Cambridge, UK,1995.

[9] H. R. Thieme, CONVERGENCE RESULTS AND A POINCAR $E$-BENDIXSON TRICHOTOMY FOR ASYMPTOTICALLY AUTONOMOUS DIFFERENTIAL EQUATIONS. J. Math. Boil., 30(1992) 755-763.

[10] H. R. Thieme, PERSISTENCE UNDER RELAXED POINT- DISSIPATIVEITY (WITH APPLICATION TO AN EPIDEMIC MODEL). Siam J. Math. Anal., 24(1992) 407-435. 
Military Technical College Kobry Elkobbah, Cairo, Egypt May 29-31,2012

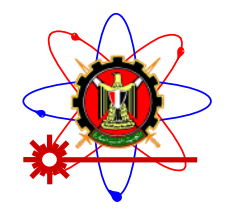

$6^{\text {th }}$ International Conference on Mathematics and Engineering Physics (ICMEP-6) 